\title{
Die Gesundheitsreform -
}

\section{(k)ein Weg zur Entlastung zukünftiger Generationen?}

Die vor der letzten Wahl gegebenen Versprechen der SPD wie auch der Unionsparteien sahen u. a. vor, eine langfristig stabile Finanzierung der GKV zu ermöglichen und damit eine finanzielle Lastverschiebung auf zukünftige Generationen zu verhindern. In diesem Beitrag wird anhand einer quantitativen Analyse aufgezeigt, dass die jüngste Gesundheitsreform nicht zu einer Entlastung zukünftiger Generationen führt und damit die Wahlversprechen nicht gehalten wurden. Zudem wird ein möglicher Weg skizziert, der zu einer nachhaltigen Entlastung zukünftiger Generationen führt. Auf diesem Weg wird der sukzessive Übergang von der derzeitigen Lohnbeitragsfinanzierung zu kapitalgedeckten Prämien in der GKV beschritten.

\section{Stefan Felder und Stefan Fetzer}

\section{Einleitung}

Die jüngst eingeführte Gesundheitsreform wird von verschiedensten Interessengruppen heftig kritisiert. Krankenkassen wie auch Leistungserbringer, Arbeitgeberverbände und Gewerkschaften, Liberale und Linke führen im Rahmen der Diskussion um die Erneuerung des Gesundheitssystem Argumente gegen die Reform an, die ebenso zahlreich sind wie die Interessen dieser Gruppen. Vergessen wird dabei häufig, warum es zu dieser Reform überhaupt gekommen ist:

Ursprüngliches Ziel der letzten rot-grünen Regierung wie auch der Union war es, die umlagefinanzierten Sozialversicherungssysteme zukunftssicher zu gestalten. Die altersmäßige Zusammensetzung der deutschen Bevölkerung wird sich nämlich binnen der nächsten fünf Dekaden aufgrund des so genannten doppelten

Alterungsprozesses (Rückgang der Geburtenzahlen einerseits und Anstieg der Lebenserwartung andererseits) massiv verändern. In der gesetzlichen Krankenversicherung (GKV) ergibt sich hieraus eine finanzielle Belastung der jungen Generationen, da diese in diesen Systemen hauptsächlich die Beiträge bezahlen, die Leistungen hingegen überwiegend von Rentnergenerationen in Anspruch genommen werden. Der medizinisch-technische Fortschritt führt zu einem zusätzlichen Anstieg der Ausgaben und damit steigenden Beitragssätzen. ${ }^{1}$ Im Ergebnis müssen künftige Generationen gegenüber lebenden Generationen einen höheren Preis für das gleiche Niveau an

Stefan Fetzer, Mitarbeiter im Wissenschaftlichen Beirats der Betrieblichen Krankenversicherung, Essen

Stefan Felder, Professor für Gesundheitsökonomie an der Universität Magdeburg und Mitglied des Wissenschaftlichen Beirats der Betrieblichen Krankenversicherung, Essen.
Gesundheitsleistungen bezahlen. Damit ist das Gebot einer nachhaltigen Finanzierung, das eine Gleichbehandlung der Generationen verlangt, verletzt. ${ }^{2}$

Vor diesem Hintergrund haben die letzte rot-grüne Regierung wie auch die damaligen Oppositionsparteien verschiedene Kommissionen (Rürup-Kommission, HerzogKommission, Nahles-Kommission) ins Leben gerufen, die Reformvorschläge für eine zukunftsfähige GKV-Finanzierung erarbeiten sollten. Das Ergebnis dieser Reformvorschläge führte - zunächst quer durch fast alle Parteien zu einen heftigen Richtungsstreit „Kopfpauschale vs. Bürgerversicherung", der sich dann im letzten Wahlkampf zuspitzte: Die Union warb für die Einführung einer Kopfpauschale, welche sie politisch opportuner Gesundheitsprämie nannte, die SPD zog mit dem Konzept der Bürgerversicherung in den Wahlkampf.

Aufgrund der gegebenen Wahlversprechen sah sich 2006 die große Koalition gezwungen, für die GKV ein Reformkonzept zu entwickeln. Die Ausgangslage mit den zwei unterschiedlichen Reformkonzepten Bürgerversicherung und Kopfpauschale ließ ganz unterschiedliche Kompromisslösungen erwarten. ${ }^{3}$ Der durch den Gesundheitsfonds gefundene Kompromiss sieht vor, 95 Prozent der GKV-Ausgaben künftig durch Beiträge und fünf Prozent durch Kopfpauschalen zu finanzieren. ${ }^{4}$ Neben dieser kombinierten Finanzierung ist weiterhin vorgesehen, mittelfristig einen Teil der GKV-Ausgaben über Steuerzuschüsse zu decken.

Angesichts der skizzierten Vorgeschichte müsste sich die Gesundheitsreform eigentlich daran messen lassen, $\mathrm{ob}$ sie zu einer langfristig stabilen und damit nachhaltigen GKV-Finanzierung beitragen kann. Im vorliegenden Beitrag wird aufgezeigt, dass die Gesundheitsreform diese Messlatte eindeutig verfehlt. Deswegen wird ein Weg zu 
einer nachhaltigen GKV-Finanzierung entworfen, nämlich durch einen Übergang zu einer kapitalgedeckten Finanzierung der GKV auf Basis altersspezifischer Prämien.

Im nächsten Abschnitt 2 wird zunächst die Nachhaltigkeit des Status quo vor der Gesundheitsreform quantifiziert. In Abschnitt 3 bzw. 4 folgen die Nachhaltigkeitsanalysen zur Gesundheitsreform bzw. zum Kapitaldekkungsmodell. Abschnitt 5 schließt mit einem Fazit.

\section{Implizite Schuld des Status quo vor der Gesundheitsreform}

Für die Quantifizierung der Nachhaltigkeit des Status quo werden zunächst die makroökonomischen Aggregate eines Basisjahres mittels alters- und geschlechtsspezifischer Profile auf die im Basisjahr lebenden Generationen verteilt. ${ }^{5}$ Auf der Ausgabenseite der GKV wird hierbei zwischen Krankengeld, ärztlichen Leistungen, zahnärztlichen Leistungen, Krankenhausleistungen, Kuren, Medikamente, Heil- und Hilfsmitteln sowie Verwaltungsausgaben unterschieden, während sich die Einnahmen der GKV aus Beiträgen von Erwerbstätigen, von Arbeitslosen und von Rentnern ergeben. Abbildung 1 zeigt die - summierten altersspezifischen Beiträge und Leistungen für das Jahr 2004, wobei ein maximales Lebensalter von 100 Jahren unterstellt ist.

Bei Betrachtung des Verlaufs der Beitragszahlungen sieht man, dass diese zwischen einem Alter von 15 und 55 kontinuierlich auf ein Niveau von knapp $3000 €$ ansteigen. Mit Renteneintritt sinken diese Pro-Kopf-Beiträge

Abbildung 1: Durchschnittliche altersspezifische Beiträge und Leistungen zwischen den Individuen und der GKV im Basisjahr 2003

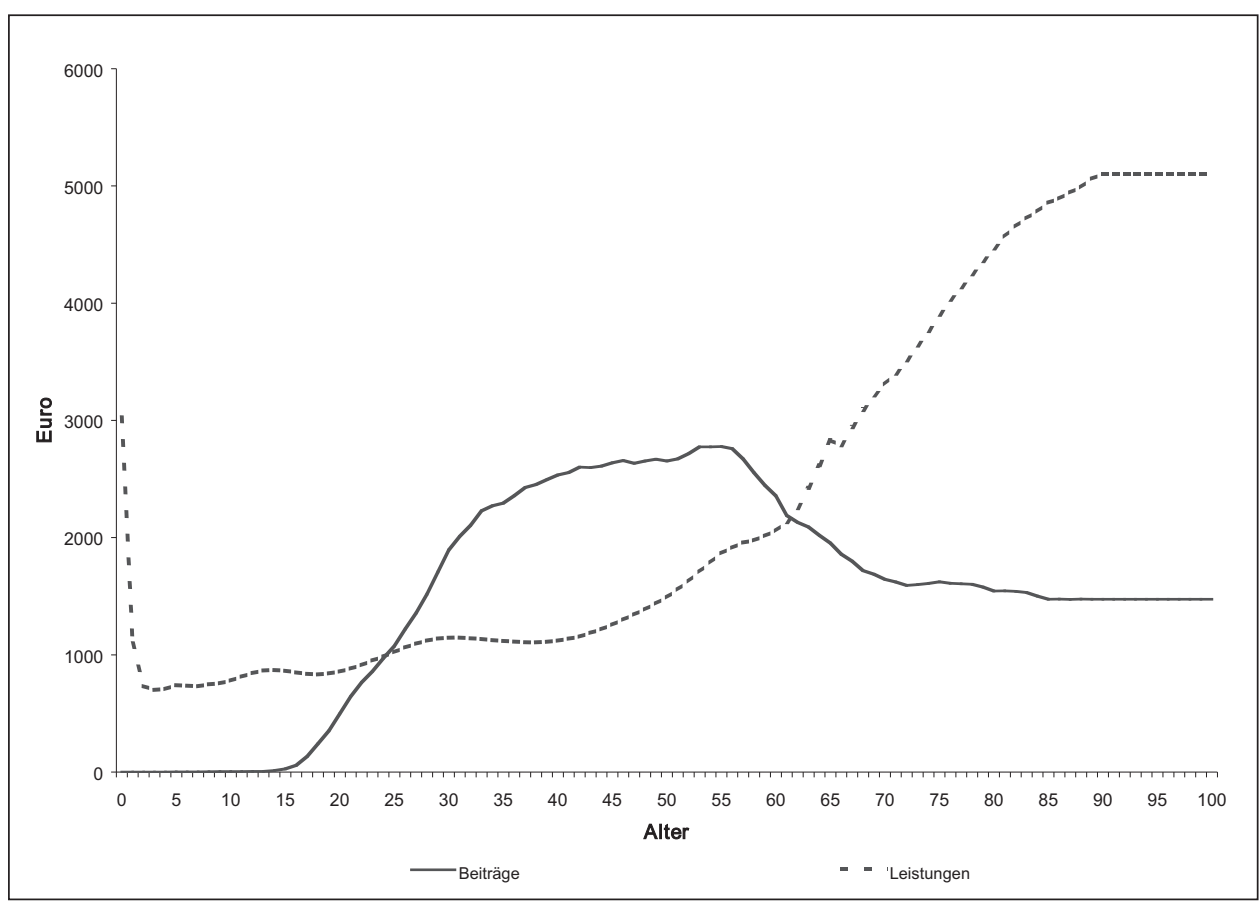

Quelle: Eigene Darstellung fast auf die Hälfte, so dass pro durchschnittlichem Rentner etwas über $1.500 € \mathrm{im}$ Jahr an Zahlungen von der GKV eingenommen werden. Ganz anders als die durchschnittlichen Beiträge entwickelt sich die Inanspruchnahme von Leistungen über den Lebenszyklus. Eine relativ hohe Inanspruchnahme erfolgt bei Geburt und in den ersten drei Lebensjahren. Danach steigt die durchschnittliche Leistungsinanspruchnahme von 700 kontinuierlich auf $2.000 €$ zum Renteneintritt an. Ab dem 60 . Lebensjahr beginnt dann ein akzelerierender Anstieg der Gesundheitsausgaben, die durchschnittlich von 2.000 auf $5.000 €$ ansteigen.

Da sich in jedem Jahr Beiträge und Leistungsinanspruchnahme die Waage halten müssen, zeichnet sich hier alles in allem klar das Bild eines größtenteils zwischen den Generationen umverteilenden, nicht verbrieften Gesellschaftsvertrags ab. Es wird dabei häufig von einem Drei-Generationenvertrag gesprochen, da bei jungen Jahrgängen (0- bis 20-Jährigen) und bei den Rentnern die durchschnittlichen Leistungen die Beiträge übersteigen, bei den Kohorten der Erwerbspersonen sich dies jedoch genau umgekehrt verhält. ${ }^{6}$

Für die Quantifizierung von Nachhaltigkeit werden in einem zweiten Schritt die in Abbildung 1 dargestellten altersspezifischen Zahlungsströme in die Zukunft fortgeschrieben. Hierbei wird für alle Einnahmenströme und für die Ausgaben Krankengeld und Verwaltungskosten eine konstante jährliche Wachstumsrate von $g=1,5$ Prozent unterstellt, welche den allgemeinen Produktivitätsfortschritt widerspiegelt. ${ }^{7}$ Für die Leistungsausgaben der GKV wird hingegen eine - ebenfalls konstante - aber höhere Wachstumsrate i. H. v. 2 Prozent, für die nächsten 40 Jahre bei der Fortschreibung angenommen, wodurch ein zukünftiges Wirken des medizinisch-technischen Fortschritts modelliert werden soll. ${ }^{8}$ Die Annahme konstanter Wachstumsraten impliziert zwar, dass die durchschnittlichen altersspezifischen Zahlungsströme mit der Zeit wachsen, das Verhältnis der Zahlungsströme unter den Kohorten eines Jahres aber unverändert bleibt. ${ }^{9}$

In einem dritten Schritt werden die fortgeschriebenen Zahlungsströme mit den in den jeweiligen zukünftigen Jahren lebenden Kohortenmitgliedern multipliziert, wodurch sich die zukünftige Beitrags- und Ausgabenentwicklung der GKV ergibt. Hierzu ist die Verwendung einer umfassenden Bevölkerungsprognose notwendig. ${ }^{10}$ 
In einem vierten Schritt werden die zukünftigen Beitragszahlungen gerade so erhöht, dass sie die Ausgaben des jeweiligen Jahres decken. Dadurch kann berechnet werden, wie sich - bei vorgegebener Ausgabenentwicklung - der durchschnittliche Beitragssatz zur GKV entwickelt. Unter den getroffenen Annahmen überschreitet dieser ausgehend von 14,3 Prozent im Jahr 2006 bereits 2020 die 16 Prozentmarke, erreicht 2030 mehr als 18 Prozent und steigt bis 2055 auf über 22 Prozent.

In einem fünften Schritt werden in jedem zukünftigen Jahr für jede Kohorte die durchschnittlichen - durch Schritt 4 angeglichenen - Beitragszahlungen mit den empfangenen Leistungen saldiert, mit den in diesem Jahr lebenden Mitgliedern der Kohorte gewichtet und auf das Basisjahr mit Hilfe eines konstant angenommenen Realzinses von $r=3$ Prozent diskontiert. Die Summe dieser Salden entspricht genau dem Betrag den alle lebenden Generationen über ihr verbleibendes Leben netto in die GKV einzahlen. Für die gewählten Parameter beträgt dieser Betrag -24,47 Prozent des BIP von 2003. Mit anderen Worten sind aufgrund der aktuellen GKV-Finanzierung lebende Generationen mit einem Betrag von 24,47 Prozent des BIP bei künftigen Generationen implizit verschuldet. ${ }^{11}$

Für den weiteren Untersuchungsgang ist es nützlich, diese Schuld noch weiter zu unterteilen. In Abbildung 1 wurde veranschaulicht, dass es sich bei der GKV bei einer jährlichen Betrachtung um einen Drei-Generationenvertrag handelt, bei dem die Nettozahlungen der erwerbstätigen Generationen die Nettotransfers der Rentnergenerationen und diejenigen der Kinder finanzieren. In dynamischer Hinsicht ergeben sich nun auf dieser Basis zwei

\section{Abbildung 2: Implizite Schuld des Status quo}

Basisjahr 2003, $g=1,5 \%, r=3 \%$, Wachstumsdifferential Leistungsausgaben 0,5\%

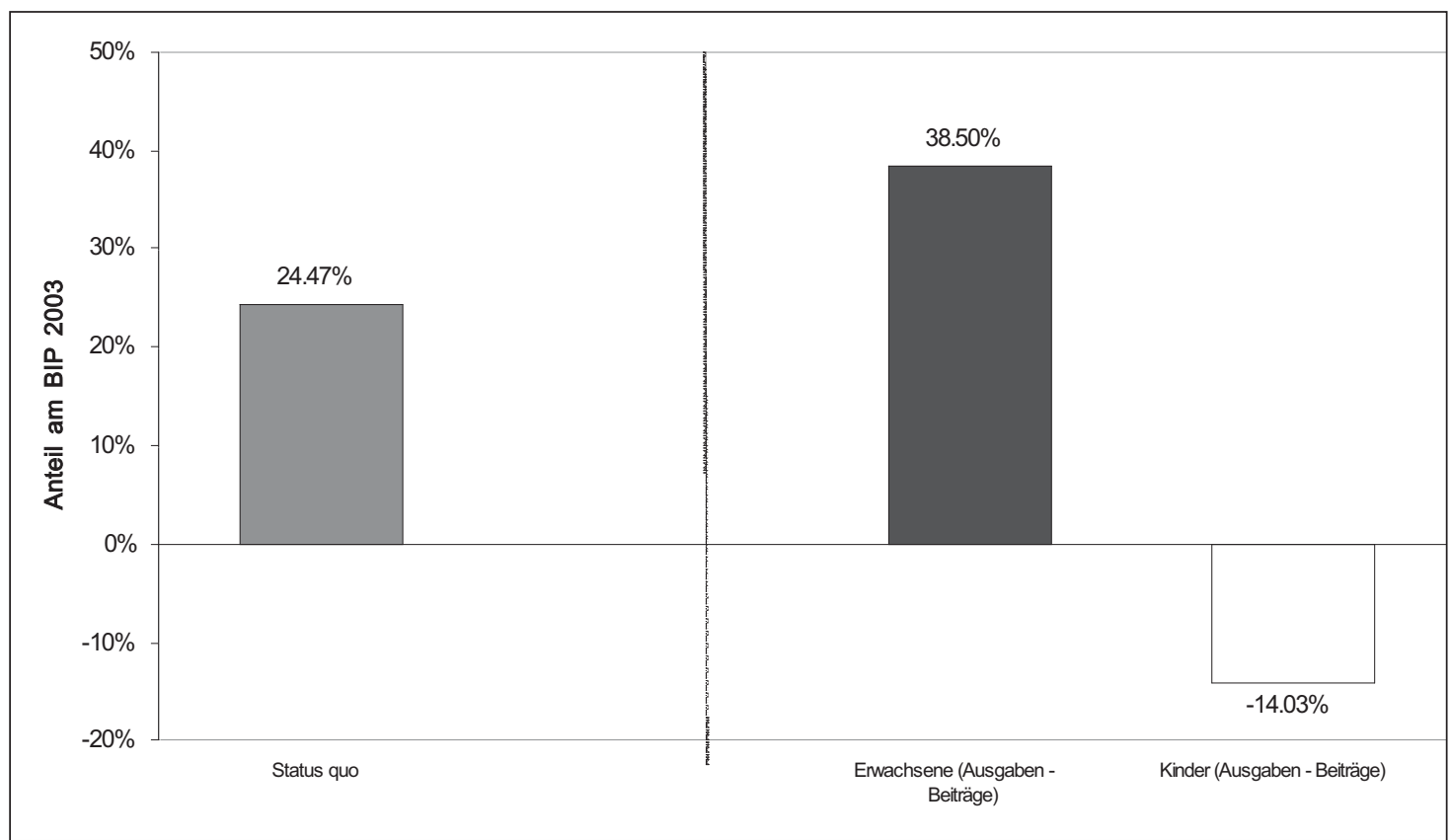

Quelle: Eigene Darstellung
Umverteilungsströme, nämlich einmal eine Umverteilung von jung zu alt zwischen Erwerbstätigen und Rentnern und einmal eine Umverteilung von alt zu jung zwischen Erwerbstätigen und Kindern. Abbildung 2 zeigt das Zustandekommen der Schuld von 24,47 Prozent des BIP durch diese Umverteilungsströme.

Der schwarze Balken spiegelt dabei die Tatsache wider, dass die zukünftigen Ausgaben der lebenden Generationen deren zukünftige Beitrags- und Kopfpauschalzahlungen bei Weitem überschreiten und so eine implizite Schuld i. H. v. 38,50 Prozent des BIP via des Generationenvertrags „Umverteilung von jung zu alt“ zukünftigen Generationen hinterlassen wird. Der weiße Balken beinhaltet den Generationenvertrag „Umverteilung von alt zu jung“. Hier müssen lebende Generationen über ihr verbleibendes Leben sehr viel Beiträge für die Finanzierung der von Kindern verursachten Ausgaben bezahlen, während bei den lebenden Generationen nur die im Basisjahr unter 17-Jährigen in den Genuss der Ausgaben dieses Generationenvertrages kommen. Insgesamt ergibt sich via Generationenvertrag „Umverteilung von alt zu jung“ ein implizites Vermögen i. H. v. 14,03 Prozent des BIP.

\section{Nachhaltigkeitswirkung der Gesundheitsreform}

Die Gesundheitsreform 2006 sieht vor, mindestens 95 Prozent der Ausgaben des Gesundheitsfonds durch Beiträge zu finanzieren. Angesichts steigender Ausgaben ist damit zu rechnen, dass der Gesundheitsfonds eher früher als später nur noch 95 Prozent der Ausgaben finanziert. Wir unterstellen daher, dass künftig fünf Prozent der

jährlichen Ausgaben durch eine Kopfpauschale finanziert werden. Des Weiteren soll bei der Nachhaltigkeitsuntersuchung der in der Gesundheitsreform 2006 geplanten partiellen Steuerfinanzierung Rechnung getragen werden. Der Steuerzuschuss wird hierzu systematisiert und richtet sich in seiner Höhe nach den GKVAusgaben für Kinder. ${ }^{12}$ Dabei wird gemäß dem Prinzip der NonAffektion von 
Abbildung 3: Implizite Schuld der Gesundheitsreform 2006

Basisjahr 2003, $g=1,5 \%, r=3 \%$, Wachstumsdifferential Leistungsausgaben $0,5 \%$

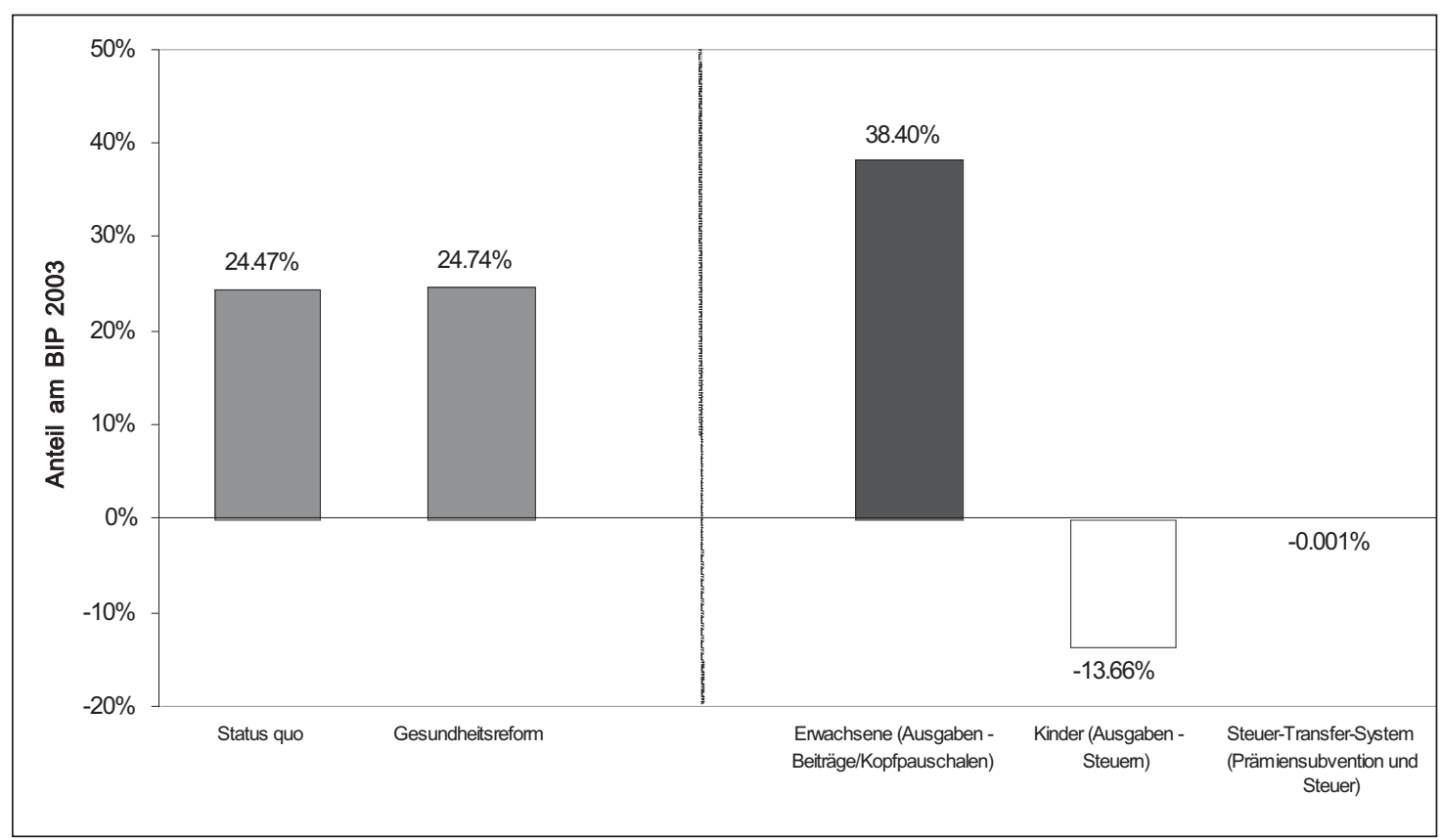

Quelle: Eigene Darstellung

Steuern angenommen, dass dieser Betrag vollständig über das allgemeine Steueraufkommen finanziert wird. ${ }^{13}$

Neben der modifizierten Finanzierungsform der zukünftigen Ausgaben wird noch die teilweise Auslagerung der Einkommensumverteilung in ein Steuer-Transfersystem berücksichtigt. Hierzu wird angenommen, dass sich ein steuerfinanzierter Subventionsbedarf ergibt, indem für jeden Haushalt in jedem zukünftigen Jahr eine Überprüfung stattfindet, ob die gezahlten Beiträge zzgl. der Kopfpauschalen, das mit dem Beitragssatz dieses Jahres gewichtete Haushaltseinkommen überschreitet. ${ }^{14}$ Dadurch kann sichergestellt werden, dass gegenüber dem Status quo reine Lohnempfänger zumindest nicht schlechter gestellt werden. ${ }^{15}$

Unter Berücksichtigung dieser Modifikationen können nun die im Abschnitt 2 beschriebenen Nachhaltigkeitsberechnungen für die Gesundheitsreform durchgeführt werden. Abbildung 3 stellt hierbei die implizite Schuld, die lebende Generationen zukünftigen aufbürden, der Gesundheitsreform dem Status quo gegenüber. Neben der gesamten impliziten Schuld dieser Reformoption, sind noch - analog zu Abbildung 2 - deren Komponenten ausgewiesen.

Offensichtlich gelingt es durch die Gesundheitsreform 2007 nicht, die Schuld zu reduzieren, die lebende Generationen zukünftigen aufbürden. Im Gegenteil, die implizite Schuld steigt ausgehend von 24,47 Prozent des BIP im Status quo vor der Reform sogar leicht auf 24,74 Prozent des BIP. Durch die Aufteilung der Schuld in die Reformbestandteile wird die Wirkungsweise der Reform ersichtlich: Der schwarze Balken beinhaltet die Schuld über den dynamische Generationenvertrag „Umverteilung von jung zu alt". Mit 38,4 Prozent des BIP zeigt sich diese Schuld geringfügig niedriger als im Status quo (38,5 Prozent). Der Grund für diesen Rückgang liegt in der Einführung von Kopfpauschalen, durch die vor allem Rentnergenerationen stärker zur Finanzierung herangezogen werden. Da bei der Gesundheitsreform nur fünf Prozent durch Kopfpauschalen finanziert werden, fällt dieser Rückgang mit 0,1 Prozentpunkten sehr gering aus. Aber auch die Wirkung einer vollständigen Finanzierung durch Kopfpauschalen wäre äußerst begrenzt und beträgt maximal rund 2 Prozentpunkte. Kopfpauschalen führen nämlich lediglich bei den Zahlungen an die GKV zu einem einheitlichen Profil, das Ausgabenprofil aus Abbildung 1 bleibt hingegen unverändert. Offensichtlich sind es die mit dem Alter sehr stark zunehmenden Ausgaben, die hauptsächlich die Schuld im Generationenvertrag „Umverteilung von jung zu alt" verursachen.

Der weiße Balken beinhaltet den nun in der Reformkomponente „Steuerfinanzierung der Kinder" organisierten Generationenvertrag „Umverteilung von alt zu jung“. Hier ergibt sich ein implizites Vermögen i. H. v. 13,66 Prozent des BIP, was gegenüber dem Vermögen im Status quo von 14,03 Prozent des BIP geringer ausfällt. Der Grund für diesen leichten Rückgang liegt darin, dass der Barwert der zukünftigen Beitragszahlungen lebender Generationen im Status quo leicht höher ist als der Barwert der zukünftigen Steuerzahlungen lebender Generationen bei der Gesundheitsreform.

Praktisch keine intergenerative Umverteilung resultiert aus dem Steuer-Transfer-Mechanismus zur Finanzierung der Prämiensubvention. Alles in allem ergibt sich durch die Gesundheitsreform also keine merkliche Nachhaltigkeitswirkung. Damit zeigt sich deutlich, dass die ursprünglich gegeben Wahlversprechen, eine zukunftsfähige, generationengerechte GKV zu etablieren, eindeutig gebrochen wurden. 


\section{Was also tun?}

Will man eine nachhaltigere Finanzierungsbasis der GKV schaffen, gibt es zunächst die Möglichkeit massiver Ausgabenkürzungen - sei es in direkter Form oder in indirekter Form, wie z.B. mittels hoher Eigenbeteiligungen. Soll hingegen ein umfassendes Leistungsspektrum an Gesundheitsleistungen auch weiterhin durch die GKV gewährleistet werden, so bietet sich ein schrittweiser Übergang auf ein Kapitalbildungsmodell an, das folgendermaßen aussehen könnte: ${ }^{16}$

Alle über 40-Jährigen verbleiben bei der Systemumstellung im bisherigen beitragsfinanzierten System der „alten“ GKV. Für alle jüngeren Jahrgänge wird in der neuen GKV von den Krankenkassen eine altersspezifische Prämie erhoben, die deren durchschnittlichen altersspezifischen Kosten entspricht (vgl. Abbildung 1). Somit kommt es zu einer sukzessiven Verdrängung des beitragsfinanzierten Systems durch das System altersspezifischer Prämien.

Allerdings steigt die altersspezifische Prämie mit zunehmendem Alter sehr stark an. Um diesem Problem zu begegnen, kommt nun die Kapitaldeckung ins Spiel: Die mit dem Alter ansteigende Prämie wird über den Lebenszyklus mit Hilfe von Ersparnissen auf individuelle Extrakonten, welche in späteren Jahren für die teilweise Finanzierung der altersspezifischen Prämie verwendet werden, geglättet. Dem Staat obliegt es hierbei, erstens sicherzustellen, dass alle Versicherten auch tatsächlich die entsprechenden Ersparnisse bilden (Zwangssparen), und zweitens den Zweck der Ersparnisse auf die Prämienfinanzierung zu beschränken (Zweckbindung).

Mit dem Übergang auf ein solches Modell sind erhebliche verteilungspolitische Konsequenzen verbunden. So übersteigen bei einkommensschwachen Gruppen die Prämienzahlungen teilweise erheblich deren bisherige Beitragszahlungen. Deshalb ist es geboten, das Modell sozial zu flankieren. Wird - analog zur Modellierung der Gesundheitsreform angenommen, dass derjenige Anteil der gesamten Prämienzahlungen eines Haushalts subventioniert wird, welcher über dem mit dem Beitragssatz gewichteten Anteil des Haushaltseinkommens liegt, so kann sicher gestellt werden, dass gegenüber dem jetzigen System reine Lohneinkommensempfänger durch den Systemüber-

Quelle: Eigene Darstellung gang nicht schlechter gestellt werden. Um die in der „alten GKV“ verbleibenden Mitglieder vor zu hohen Beiträge zu schützen, wird weiterhin angenommen, dass die Ausgaben, welche die Beitragseinnahmen dieser Mitglieder im Status quo überschreiten, über das allgemeine Steueraufkommen finanziert werden.

In Abbildung 4 ist die implizite Schuld dieser Reformoption dem Status quo gegenübergestellt. Wie sich zeigt, kann die Schuld von 24,47 auf 4,26 Prozent des BIP gesenkt werden, wenn diese Reform eingeführt werden sollte. Um diese Wirkung besser zu ergründen, sind auch hier die impliziten Schulden der einzelnen Reformkomponenten dargestellt.

Die einzelnen Komponenten dieser Reform umfassen hier erstens die Nettozahlungen der verbleibenden lebenden Generationen in das alte System der GKV, also den Barwert aller Ausgaben abzüglich der zu leistenden Beitragszahlungen für alle im Jahr 2007 über 40-Jährige. Da die von diesen Generationen verursachten zukünftigen Ausgaben deren Beitragszahlungen bei Weitem übersteigen (vgl. hierzu Abbildung 1), verwundert es nicht, dass hier zunächst eine sehr hohe implizite Schuld i. H. v. 47,45 Prozent des BIP entsteht, die durch zusätzliche Steuerzahlungen abgegolten werden muss. Weil solche Steuerzahlungen aber hauptsächlich durch die heute lebenden Generationen zu leisten sind, müssen diese in großem Umfang die „alte“ GKV subventionieren. Damit ergibt sich als zweite Komponente der impliziten Schuld dieses Modells eine negative Schuld i.H.v. -41,60 Prozent des BIP. Beide Komponenten zusammengenommen geben darüber Auskunft, dass durch dieses System der „alten" GKV zukünftigen Generationen eine Schuld i.H.v. 5,85 Prozent des BIP überlassen wird, welche diese durch zusätzliche Steuern zu finanzieren haben.

Abbildung 4: Implizite Schuld des Kapitaldeckungsmodells

Basisjahr 2003, g=1,5\%, $r=3 \%$, Wachstumsdifferential Leistungsausgaben 0,5\%

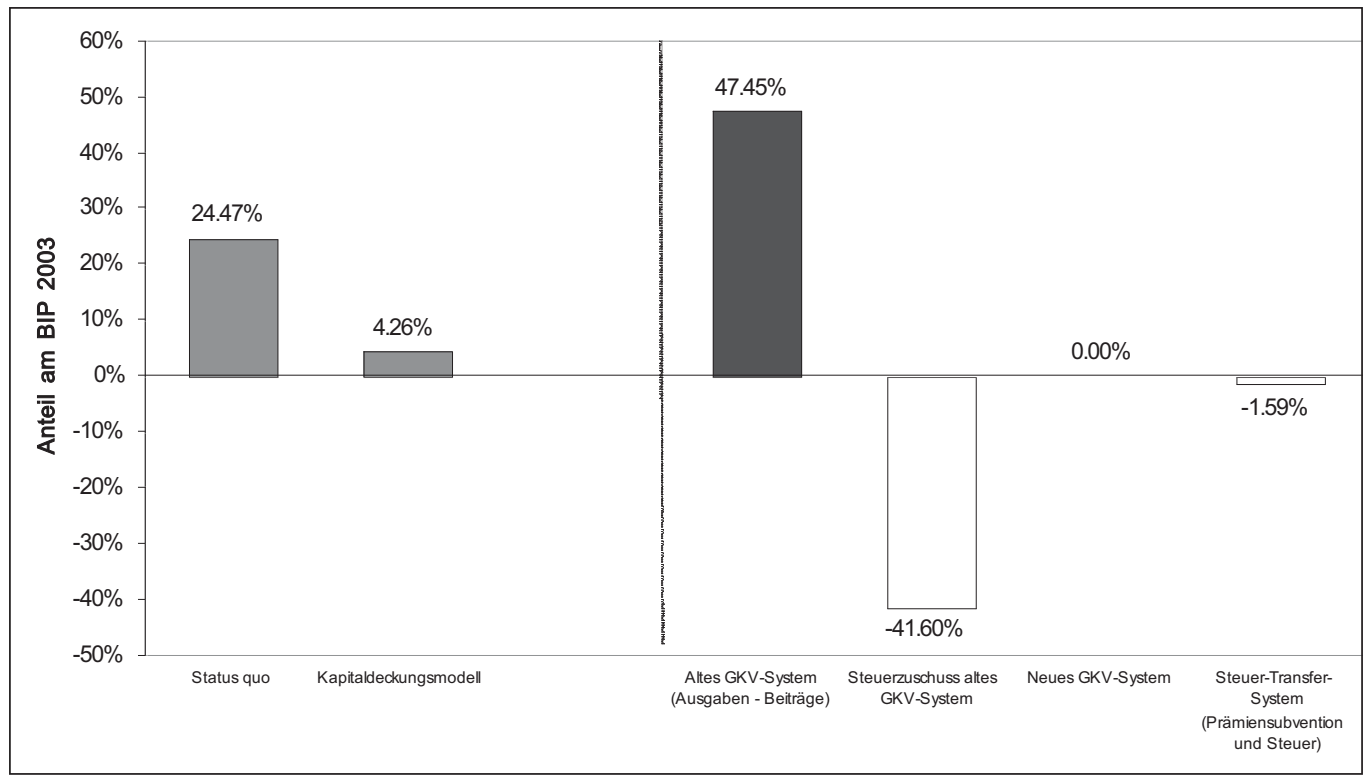


Kernstück dieses Modells sind aber sicherlich die geglätteten Prämien für im Jahr 2007 unter 40-Jährige. Da sich für jeden Jahrgang im Erwartungswert zukünftige GKV-Einnahmen und -Ausgaben entsprechen, resultiert hier in der dritten Komponente keine Schuld. Die vierte Komponente zeigt die Schuld des Steuer-Transfer-Mechanismus, welcher für die Subvention dieser Prämien implementiert wird. Wie sich zeigt, ist diese Schuld negativ und beträgt $-1,59$ Prozent des BIP. ${ }^{17}$

Den „Preis“ für diese doch merkliche Entlastung zukünftiger Generationen müssen aber - bei gleichem angenommenem Leistungsniveau - zwangsläufig lebende Generationen bezahlen. In dieser Reformoption besteht der „Preis“ v. a. aus zusätzlichen Steuerzahlungen, welche für die Subventionierung der „alten“ GKV-Mitglieder und derjenigen Teile der Gesellschaft, die sich die Prämien nicht leisten können, aufzuwenden sind.

\section{Fazit}

Im vorliegenden Beitrag wurde untersucht, inwiefern es gelingen kann, die GKV generationengerechter, d.h. nachhaltiger zu finanzieren. Dabei wurde als erstes aufgezeigt, dass in einer beitragsfinanzierten GKV lebende Generationen zukünftigen Generationen implizite Zahlungsverpflichtungen von knapp einem Fünftel der aktuellen gesamten Jahreswirtschaftsleistung hinterlassen. Durch die Gesundheitsreform ist es den Koalitionsparteien CDU/CSU und SPD nicht gelungen, dieses Problem auch nur annähernd anzugehen. Dies ist insofern auch nicht verwunderlich, als dass durch diese Reform die altersmäßigen Durchschnittszahlungen fast gar nicht verändert werden und damit die sich im System vollziehenden Umverteilungen zwischen den Generationen weitestgehend erhalten bleiben.

Wie die Ergebnisse des Kapitaldeckungsmodells gezeigt haben, kann der nicht nachhaltige Zustand in der GKV erheblich verbessert werden, wenn diese - bei entsprechenden sozialpolitischen Ausgleichszahlungen - sukzessive auf eine altersspezifische Prämienkalkulation umgestellt wird. Durch die gleichzeitige Einführung von Kapitalrückstellungen kann zudem erreicht werden, dass die Prämie über den Lebenszyklus der Versicherten geglättet wird, womit Versicherte in hohem Alter vor extrem hohen Prämien geschützt werden können.

Allerdings wird die Schuld in der GKV, welche lebende zukünftigen Generationen hinterlassen, durch die Maßnahmen des Kapitaldeckungsmodells nur zu vier Fünftel abgebaut. Um eine vollständige nachhaltige Finanzierung der GKV zu erreichen, müssen wohl ausgabenseitige Maßnahmen, wie z.B. eine Kürzung des Leistungskataloges oder der Ausbau von Selbstbeteiligungen, ergriffen werden. Ansonsten bleibt hier die wage Hoffnung, dass die mit der Gesundheitsreform 2006 gewollte Intensivierung des Wettbewerbs auf den Gesundheitsmärkten auch wirklich in Gang kommt und Effizienzreserven erschließt, welche zu spürbaren Kostensenkungen auf der Leistungsseite führen.

\section{Literatur}

Auerbach, A., J. Gokhale and L. Kotlikoff (1991), Generational Accounts: A Meaningful Alternative to Deficit Accounting, in: Bradford, D. (Hrsg.), Tax Policy an the Ecoconmy, 5, Cambridge, 55-110.

Auerbach, A., J. Gokhale and L. Kotlikoff (1992), Generational Accounting: A New Approach for Understanding the Effects of Fiscal Policy on Saving, Scandinavian Journal of Economics, 94, 303-318.

Auerbach, A., J. Gokhale and L. Kotlikoff (1994), Generational Accounting: A Meaningful Way to Evaluate Fiscal Policy, Journal of Economic Perspectives, 8, 73-94.

BMGS - Bundesministerium für Gesundheit und Soziales (2006), Die neue Gesundheitsversicherung - Die wesentlichen Veränderungen durch die Gesundheitsreform 2006, Stand 25.10.2006, www.die-gesundheitsreform.de.

Bonin, H. (2001), Generational Accounting: Theory and Application, Berlin. Breyer, F. und S. Felder, (2004), Life Expectancy and Health Care Expenditures: A New Calculation for Germany Using the Costs of Dying, DIW Discussion Paper 452, Berlin.

Breyer, F. und V. Ulrich, (2000), Gesundheitsausgaben, Alter und medizinischer Fortschritt: Eine Regressionsanalyse, Jahrbücher für Nationalökonomie und Statistik, 220, 1-17.

Felder, S. (1997), Vom „Deficit Accounting“ zum „Generational Accounting“: Eine Anwendung für die Schweiz, Zeitschrift für Volkswirtschaft und Statistik, 133 (3), 497-512.

Fetzer, S. (2006), Zur nachhaltigen Finanzierung des gesetzlichen Gesundheitssystems, Frankfurt.

Gelijns, A.C. and N. Rosenberg (1994), The Dynamics of Technological Change in Medicine, Health Affairs, 13, 28-46.

Knappe, E. und U. Rachold (1997), Demographischer Wandel und Gesetzliche Krankenversicherung, in: Knappe, E. und A. Winkler (Hrsg.), Sozialstaat im Umbruch, Frankfurt, 91-118.

Kotlikoff, L. und C. Hagist (2005), Who's going broke? Rising health care costs in ten OECD countries, NBER Working Paper, 11833.

Newhouse, J. (1992), Medical Care Costs: How Much Welfare Loss?, Journal of Economic Perspectives, 6, 3-21.

Oberender, P, S. Felder, V. Ulrich, U. Schneider, A. Werblow und J. Zerth (2006), Bayrether Versichertenmodell, Bayreuth.

Okunade, A. und V. Murthy, (2002), Technology as a ,major driver' of health care costs: a cointegration analysis of the Newhouse conjecture, Journal of Health Economics, 21, 147-159.

Raffelhüschen, B. (1999), Generational Accounting: Method, Data, and Limitations, European Economy, Reports and Studies, 6, 17-28.

Schulenburg, J. Graf von der (1989), Gesundheitswesen (Krankenversicherung) und demographische Evolution, in: Recktenwald, H. (Hrsg.), Der Rückgang der Geburten - Folgen auf längere Sicht : ein Symposion der Akademie der Wissenschaften und der Literatur, Mainz, 22. - 23. 6. 1988, 279-297.

Statistisches Bundesamt (2003), Bevölkerung Deutschlands bis 2050 - 10 . koordinierte Bevölkerungsvorausberechnung, CD-ROM, Wiesbaden.

Statistisches Bundesamt (2005), Einkommens- und Verbrauchsstichprobe 2003, Wiesbaden.

SVR - Sachverständigenrat zur Begutachtung der gesamtwirtschaftlichen Entwicklung (2004), Erfolge im Ausland - Herausforderungen im Inland, Jahresgutachten 2004/2005, Stuttgart.

Ulrich, V., S. Felder, U. Schneider und A. Werblow (2005), Stärkung der Nachhaltigkeit in der Finanzierung des Versicherungsschutzes der GKVVersicherten, Gutachten im Auftrag der Kassenärztlichen Bundesvereinigung (KBV).

WCED - World Commission on Environment and Development (1987), Our Common Future, Oxford University Press.

\section{Fußnoten}

1 Vor allem von Arbeitgeberseite wird hier häufig das Argument vorgebracht, dass bei einer weiteren Steigerung der Beitragssätze und damit der Lohnnebenkosten die in Deutschland produzierten Güter kostenintensiver hergestellt werden müssen. Dies beeinträchtige die internationale Wettbewerbsfähigkeit deutscher Unternehmen und lasse einen Anstieg der Arbeitslosigkeit befürchten. Zumindest für 
den Niedriglohnsektor erscheint dieses Argument plausibel, da die Nettolöhne aufgrund tariflicher Regelungen aber auch aufgrund der Ausgestaltung der staatlichen Existenzsicherung via Sozialhilfe u. ä. nach unten begrenzt sind.

2 Die Brundtland-Kommission (benannt nach dem Namen der Vorsitzenden der Kommission, der ehemaligen norwegischen Ministerpräsidentin Gro Harlem Brundtland) definiert Nachhaltigkeit wie folgt: "Sustainable development is development that meets the needs of present without compromising the ability of future generations to meet their own needs.", vgl. WCED (1987).

3 Z.B. können beide Modelle konsequent zu einer so genannten Bürgerpauschale kombiniert werden, für deren Einführung z.B. der Sachverständigenrat zur Begutachtung der gesamtwirtschaftlichen Entwicklung (SVR) in seinem Jahresgutachten 2004/2005 plädiert, vgl. SVR (2004).

4 So steht in einer Kurzinformation zur Gesundheitsreform des BMGS (2006): „Eine Überforderung der Kassen und ihrer Mitglieder wird schon im Ansatz vermieden, indem gesetzlich festgelegt wird, dass der Gesundheitsfonds immer mindestens 95 Prozent der Ausgaben aller Kassen deckt. Wird dieser Schwellenwert erreicht, werden aufgrund einer gesetzlichen Anpassungsregelung die prozentualen Beiträge aller Versicherten und der Arbeitgeber angehoben."

5 Die hier zugrundeliegende Methode ist eine Modifikation der Generationenbilanzierung, die Anfang der 1990er Jahre von Auerbach, Gokhale und Kotlikoff $(1991,1992,1994)$ entwickelt wurde. Die bislang umfassendste Arbeit ist eine Vergleichsstudie im Auftrag der Europäischen Kommission, vgl. hierzu Raffelhüschen (1999). Die hier verwendete modifizierte Version wurde von Felder (1997) entwickelt und kam für die Nachhaltigkeitsanalyse von verschiedenen Gesundheitsreformoptionen bereits in einem Gutachten für die Kassenärztliche Bundesvereinigung zur Anwendung, vgl. hierzu Ulrich et al. (2005). Für die folgende Vorgehensweise sowie die verwendeten Daten vgl. Fetzer (2006)

6 Vgl. hierzu auch Schulenburg (1989) oder Knappe und Rachold (1997).

7 Daneben wird für die Beiträge der Rentner und der Rentenversicherung angenommen, dass diese um die in den letzten Jahren beschlossenen Rentenkürzungen (wie z.B. Nachhaltigkeitsfaktor) langfristig sinken.

8 Newhouse (1992) macht für die überproportionale Gesundheitsausgabensteigerung der Vergangenheit den medizinisch-technischen Fortschritt, bei dem kostenverursachende Produktinnovationen kostensenkende Prozessinnovationen dominieren, verantwortlich Empirischen Nachweis findet diese These beispielsweise durch Gelijns und Rosenberg (1994) und Okunade und Murthy (2002) für die meisten OECD-Länder. Das für die folgenden Berechnungen angenommene Wachstumsdifferential i. H. v. 0,5 Prozent kann noch als relativ moderate kostensteigernde Wirkung des medizinisch-technischen Fortschritts angesehen werden, denn für Deutschland kommen anhand empirischer Studien Breyer und Ulrich (2000) auf ein Wachstumsdifferential von etwa einem Prozentpunkt, Kotlikoff und Hagist (2005) sogar auf 1,7 Prozentpunkte. Eine Begrenzung des Wirkungszeitraums (hier auf 40 Jahre) ist insofern notwendig, als dass es sonst langfristig zu dem widersprüchlichen Resultat kommen könnte, dass die Gesundheitsausgaben über dem BIP liegen.

9 Diese Annahme ist für Gesundheitsausgaben nicht ganz unproblematisch. Denn immer noch sehr strittig sind in der gesundheitsökonomischen Literatur die Konsequenzen einer verlängerten Lebenserwartung auf die Gesundheitsleistungsinanspruchnahme. Allerdings zeigen Breyer und Felder (2004) sowie Fetzer (2006), dass bei langfristigen Betrachtungshorizonten die unterstellte Konsequenz aus einer verlängerten Lebenserwartung gegenüber der Berücksichtung des medi- zinisch-technischen Fortschritts von eher untergeordneter Bedeutung für das quantitative Ausmaß der Ergebnisse ist.

10 Im vorliegenden Fall wurde als Basis die mittlere Variante der 10. koordinierten Bevölkerungsprojektion des Statistischen Bundesamtes verwendet, vgl. hierzu Statistisches Bundesamt (2003). Allerdings ist bei der hier verwendeten Bevölkerungsprojektion, die auf Basis eines Modells von Bonin (2002) gewonnen wurde, der Betrachtungshorizont mit 100 Jahren länger als die Bevölkerungsprojektion des Statistischen Bundesamtes, welche bis 2050 erhältlich ist.

11 Bei der herkömmlichen Methode der Generationenbilanzierung findet bei der Berechnung der impliziten Nettozahlungen, die hier Generationenkonten genannt werden, keine jährliche Angleichung der Beitragszahlungen an die Ausgabenentwicklung statt (es wird also auf Schritt vier verzichtet). Die sich dann ergebende implizite Schuld wird hingegen nicht nur durch alle im Basisjahr lebenden, sondern auch durch alle zukünftig lebenden Generationen verursacht und ist nichts anderes als der Barwert aller zukünftigen GKV-Defizite. Im Prinzip weist diese Schuld dann denjenigen Anpassungsbedarf aus, der über einen (zumindest theoretisch) unendlichen Betrachtungshorizont in der GKV resultiert.

12 Diese Systematisierung ist allerdings nicht im Entwurf der Gesundheitsreform 2006 enthalten. Vielmehr bekommt man den Eindruck, dass der Steuerzuschuss in den Gesundheitspool sich eher an der aktuellen konjunkturellen Lage orientiert. Anfang des Jahres 2006 wurde der Steuerzuschuss aus der erhöhten Tabaksteuer erst einmal gestrichen. Nachdem abzusehen war, dass die Steuereinnahmen in diesem Jahr höher als geplant ausfallen, kam die Regierung hingegen wieder auf die Idee, diese teilweise zur Finanzierung des Gesundheitsfonds zu verwenden.

13 Damit wird das geltende Umlageverfahren in der GKV auf die zusätzlichen Steuer-Transferzahlungen übertragen, die sich bei einer Reform ergeben.

14 Dieser Steuer-Transfermechanismus orientiert sich an älteren Kopfpauschalmodellen. Die Überprüfung, ob die gezahlten Beiträge zzgl. der Kopfpauschalen, das Haushaltseinkommen übersteigen geschieht anhand von Daten aus der Einkommens- und Verbrauchsstichprobe (EVS) 2003 des Statistischen Bundesamts (2005). Um die den Nachhaltigkeitsberechnungen zugrunde liegende Dynamik zu modellieren, wird weiterhin ein Wachstum des Haushaltseinkommens mit der Rate g und eine Entwicklung der Überforderungsgrenze gemäß der Beitragssatzentwicklung des Status quo unterstellt.

15 Dieses Vorgehen weicht vom Gesundheitsreformbeschluss ab, bei dem vorgesehen ist, dass ein Abgleich der Überforderung und auch die Umverteilung auf Kassenebene durchgeführt wird. Es ist allerdings mehr als fraglich, ob eine Umverteilung innerhalb der Krankenkasse überhaupt praktikabel ist. Da hier ja aber primär die Nachhaltigkeitswirkung der Reform modelliert werden soll, erscheint ein Transfermechanismus, bei dem - bezogen auf das beitragspflichtige Einkommen - keine Schlechterstellung gegenüber dem Status quo vor der Reform stattfindet, wesentlich plausibler zu sein als ein nur schwer hinsichtlich seiner Gesamtwirkung quantifizierbarer kassenspezifischer sozialer Ausgleich. Zudem gewährleistet das hier gewählte Vorgehen auch die Vergleichbarkeit mit der folgenden Reformoption.

16 Das hier im Folgenden diskutierte Kapitaldeckungsmodell orientiert sich im Wesentlichem am so genannten Bayreuther Modell von Oberender et. al (2006).

17 Ein Faktum, welches darauf zurückzuführen ist, dass zukünftige Generationen erst einmal sehr wenig verdienen und damit erst einmal in den Genuss der Prämiensubvention kommen. Ebenso sind deren durchschnittliche altersspezifischen Steuerzahlungen am Anfang des Lebenszyklus auch sehr niedrig. 